\title{
Design of Robotic Arm for Disaster Management
}

\author{
Naseem Rao \\ Assistant Professor, CSE Department, Hamdard University, Delhi, India
}

\begin{abstract}
In this paper, Design of Robotic Arm for Disaster Management is proposed. The Robotic Arm is to perform various functions by the mean of employing process or sequence of programmed operation with little or no human labor using Ad-hoc techniques. The goal of less or no probability of error with real time management is achieved. Easy operation by use of smartphones is achieved. Elimination of the stressed work by the humans on the systematic working to achieve desired production in the industry is made. By developing an autonomous nature of the system, the same is made a reliable one.
\end{abstract}

Keywords: Robotic Arm; ROVER-BOT;MCU; MPU; Automatic

\section{INTRODUCTION}

The Robotic Arm is a lightweight and expandable robot that can operate in extreme conditions where the human presence or availability is not possible due to various reasons. It can be also termed as an unmanned vehicle because it can be remotely operated by game-style hand controller. This robot can also be used as warfighters and first responders to carry out dangerous missions in high-threat battlefield scenarios to minimize the human casualties. This particular project actually comprises of two parts where the base part is for mobility and upper part is a robotic hand that performs the functions given to it wirelessly by the controller. It is a portable and easy-to-use modular design. It can be deployed in less than two minutes and operates under all weather conditions.

\section{PROPOSED ARCHITECTURE}

This work is able to successfully accomplish the defined functionality. A sample robot which can rotate, magnetize an object, lower and raise its arm, by being controlled by the microcontroller is built successfully. The development board is soldered and it used the required procedure for the correct operation of the controller. The development board has been interfaced to the stepper, servo \& dc motors such that the anthropomorphic like structure can be controlled from the buttons at the base of the structure (robotic arm). When signal come to robotic arm, it will activated and then it searching for the code object in storage area when it get confirmation of availability thus it call rover to collect and dispatch that object to its destination of call at the instant of getting signal inbuilt program in arduino controller activate and check signal status incoming signal on terminal via Node MCU module thus programmed the DC motor with fixed degree which have been place in programming for particular objects, end effecter as a claw made up of servo geared motor and spar gear assembly with L293d motor driver.

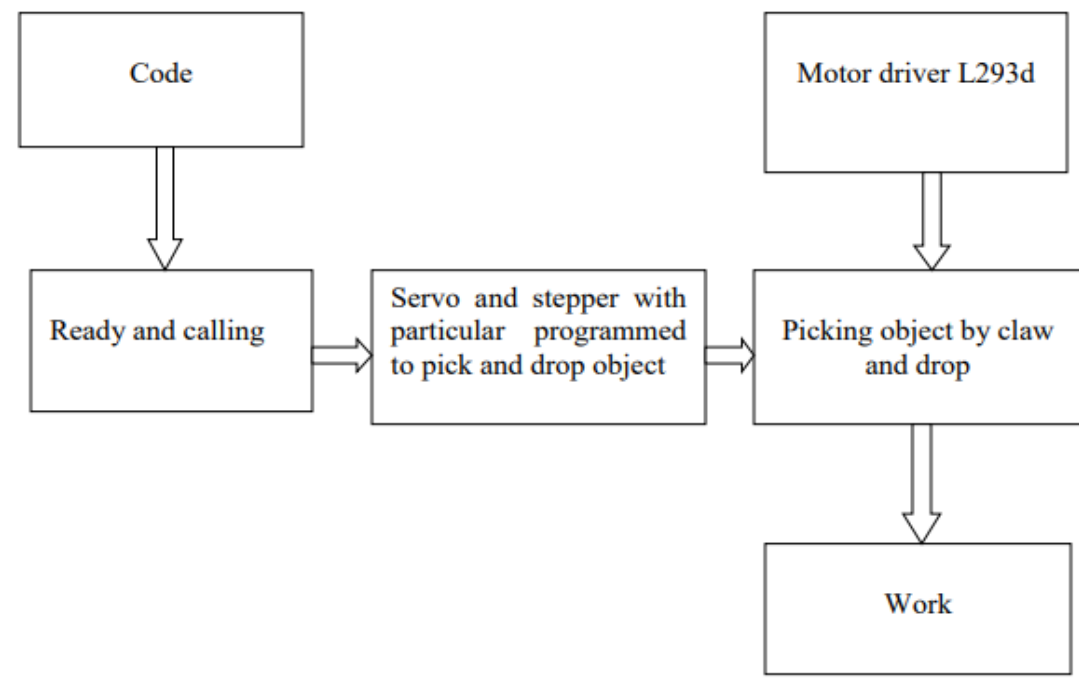

Fig 1: Flowchart for the working of Robotic arm 


\section{International Advanced Research Journal in Science, Engineering and Technology}

Vol. 6, Issue 7, July 2019

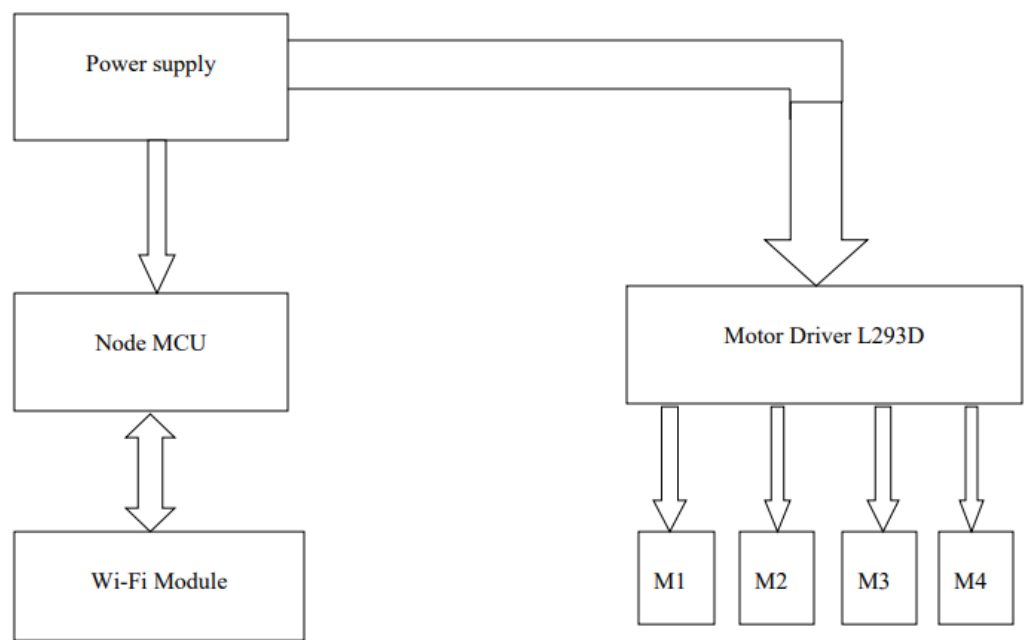

Fig 2: Proposed methodology for the working of Rover or vehicle used

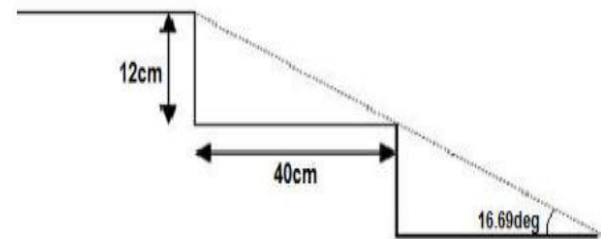

Fig 3: Tilt Angle \& Wheel Base

III.

\section{CONCLUSION}

In this work, the Robotic Arm is to perform various functions by the mean of employing process or sequence of programmed operation with little or no human labour using Ad-hoc techniques. The goal of less or no probability of error with real time management is achieved. Easy operation by use of smartphones is achieved. Elimination of the stressed work by the humans on the systematic working to achieve desired production in the industry is made. By developing an autonomous nature of the system, the same is made a reliable one. A solution for the simultaneous accomplishment of assembling work done in the industries by the means of single computes has not only provided ease in operation but also provides autocratic nature for monitoring purpose is designed.

\section{REFERENCES}

[1]. Hong-an Yang, Luis Carlos Velasco Rojas*, Changkai Xia, Qiang Guo, School of Mechanical Engineering, Northwestern Polytechnic University, Xi'an, China, Dynamic Rocker-Bogie: A Stability Enhancement for High- Speed Traversal- Vol. 3, No. 3, September 2014, pp. 212 220 ISSN: 2089-4856.

[2]. R.E. Moore, Interval analysis (Englewood Cliffs, NJ: Prentice-Hall, 1966).

[3]. Brooks Thomas; Graham Gold; Nick Sertic; DARK ROVER ROCKER-BOGIE OPTIMIZATION DESIGN, The University of British Columbia, Project Number 1076 Jany 18, 2011. Jiewen Zheng, Zhengbo Zhang,Taihu Wu, Guang Zhang, Emerging Wearable Medical Devices towards Personalized Healthcare, Conference: Proceedings of the 8th International Conference on Body Area Networks, September 2013.

[4]. S Tanweer, A Mobin, A Alam, "Environmental Noise Classification using LDA, QDA and ANN Methods", Indian Journal of Science and Technology, Vol 9(33), September 2016 ,ISSN (Print) : 0974-6846 ISSN (Online) : 0974-5645

[5]. Inderpreet Singh, Arrvindh Shriraman, Wilson WL Fung, Mike O'Connor, Tor M Aamodt, Cache coherence for GPU architectures, IEEE 19th International Symposium on High Performance Computer Architecture (HPCA2013), 2013.

[6]. Ramzi A. Haraty, Mohamad Dimishkieh,Mehedi Masud, An Enhanced k-Means Clustering Algorithm for Pattern Discovery in Healthcare Data, International Journal of Distributed Sensor Networks vol. 11,June 2015.

[7]. Analysis of K-Means and K-Medoids Algorithm For Big Data, Procedia Computer Science, Volume 78, 2016, Pages 507-512 in 1 International Conference on Information Security \& Privacy ,2015.

[8]. Bottou L.,Bengio Y.Tesauro and G., Touretzky D.Convergence properties of the k-means algorithms Advances in Neural Information Processing Systems 7New York, NY, USAMIT,1995.

[9]. The cerebellum microcontroller. http://www.roboticsclub.org/ cereb.

[10]. P. Coppin, A. Morrissey, M. Wagner, M. Vincent, G. Thomas, Big signal: information interaction for public telerobotic exploration, in: Proceedings of the Workshop on Current Challenges in Internet Robotics, ICRA, 1999.

[11]. T. Estier, Y. Crausaz, B. Merminod, M. Lauria, R. Piguet, R. Siegwart, An innovative space rover with extended climbing abilities, in: Proceedings of the Space and Robotics 2000, Albuquerque, USA, February 27-March 2, 2000.

[12]. M. Fujita, H. Kitano, Development of an autonomous quadruped robot for robot entertainment, Autonomous Robots Journal 5 (1998).

[13]. I. Horswill, Visual collision avoidance by segmentation, in: Proceedings of the IEEE/RSJ International Conference on Intelligent Robots and Systems, 1994, pp. 902-909. 